\title{
Mobile Health Services in Saudi Arabia-Challenges and Opportunities
}

\author{
Dr. Amr Jadi \\ Department of Computer Science and Information \\ College of Computer Science and Engineering \\ University of Ha'il, Ha'il \\ Saudi Arabia
}

\begin{abstract}
In this work, the mobile health services (MHS) approach has been introduced to encourage locals with different educational backgrounds. This work intends to minimize personal interaction hours between patients and doctors in a real-time healthcare environment. The increasing number of pilgrims to Saudi Arabia (SA) demands such an arrangement for the benefit of both people and service provider authorities. Especially dealing with the patients visiting at the time of Ramadan is going to be a challenging task for the authorities and healthcare service providers if some kind of virus spreads in the Kingdom. The recent Corona virus threat is making most of the people panic and almost all the countries in the world are feeling the heat to tackle such a scenario. Due to a famous pilgrim destination, dealing the visitor's flow is always a challenging task. Therefore, the proposed MHS uses the latest applications of neural networks (NN), artificial intelligence (AI), bigdata (BD) and predictive data analytics (PDA) for improving the performance of healthcare operations. At the initial stage of this research, the risk prediction and mitigation process of various events have seen an accuracy of $95 \%$. Applications of AI and BD are being extensively used to upgrade the patient records and information at a faster rate to enhance the overall performance of healthcare services.
\end{abstract}

\section{Keywords-m-Health; IoT; Saudi Hospitals; challenges}

\section{INTRODUCTION}

The mobile health (m-health) is popularly used as a support system for public health, which is supported by mobile devices to establish faster communication. Not only mobiles, but tablet computers, smartwatches, and PDAs (personal digital assistants) are used for the collection of data/information in health services [1]. The services of $\mathrm{m}$ health are taking rapid growth in the region of Saudi Arabia (SA) for quite a long time to address a wide range of solutions for various medical problems. These services are extensively using information and communication technologies (ICT) to obtain information in one place and apply the same when it is necessary [2]. These solutions proved to be very useful and potential enough to provide immediate relief for the patients with sudden changes in their health conditions due to uninterrupted monitoring qualities they have with these devices. They can extend the treatment to thousands of patients and improve the patient's condition at a time using ICT technologies [3]. Presently, the Kingdom of Saudi Arabia (KSA) is encouraging to implement new strategies for its Vision 2030 on citizen's wellness and to improve the healthcare activities. In recent times, Al-Anzi working in this area of $\mathrm{m}$-health to understand, how $\mathrm{m}$-Health can bring better results for the society of SA and help the students develop an application by which a lot of information can be propagated among the people of SA [4]. The role of health information systems was explored by Borycki et al. suggested that these technologies are helping to improve the quality and safety of the information for patients towards making any kind of health-related decisions [5]. The role of these systems to identify different types of unsafe health practices and allows considering the ways to engage with these unsafe practices in an effective manner.

A lot of work is being carried out in Saudi Arabian hospitals to use information technology (IT) and in a study, Al-Harbi tried to understand the motivation, barriers, and benefits at King Abdul-Aziz Medical City (KAMC) [6]. The results from this work summarized that healthcare providers have been constantly benefited from the use of IT, and they helped the patients and doctors to keep constant monitoring over the illness. Paules Ciprés introduced a cloud computingbased KAU-Health approach for establishing a real-time interaction for obtaining critical information for crucial decision making on an immediate basis [7]. The introduction of e-Health in KSA helps the citizens towards the personalization of information for alternative diagnoses from the viewpoint of a citizen. Also, this helps obtain the improved version of information from the latest medical journals, associations, etc. from the viewpoint of a professional of healthcare. An attempt is made to create a web-based interactive diabetes registry by Al-Rubeaan $e t$ al. in SA for the usage of healthcare management and planning [8]. The geographic information system (GIS) of the registry helps in producing different types of maps with associated diseases. The Saudi National Diabetes Registry (SNDR) helps to provide needful information for the health planners and helps in making informed decisions. Similar work has been carried out in Basra, Iran by designing and implementing a mobile diabetes management system by Istepanian et al. [9]. This method claimed to provide exemplary results with effective treatment facilities for the patient's improved healthcare delivery at the conflict regions.

A review carried out on m-Health by Silva et al. studied different scientific developments, breakthroughs and different types of open issues [10]. Different deliverables of m-Health are discussed in this paper highlighted different types of 
geographical, temporal and organizational barriers. A social robotic children diabetes management and an education system for diabetic kids of KSA were introduced by Alotaibi and Choudhury using Aisoy1 v5 robot along with m-Health technologies [11]. These arrangements are aimed to help the kids with diabetes to improve and empower the information related to diabetes and different techniques involved to manage the disease. Later, Almoliri et al. explained the mhealth with the context of the internet of things (IoT) such as compactness, IP connectivity, security, and power consumption [12]. Different types of health conditions (such as blood pressure, ECG, blood sugar, etc.) and issues related to confidentiality, privacy, and security are discussed in this work. An application for Android and iPhones (iTeethey ${ }^{\mathrm{TM}}$ ) was introduced by AlKlayb et al. for educating the mothers of below six years of age [13]. This experiment was carried out in two areas of SA with satisfactory results and improvements in the awareness of different types of health issues. The usage of mobile-based applications seems to be an easier way of learning for homemakers and even to working mothers. A similar attempt to design a mobile app was made by Elfaki and Alotaibi to deal with Alzheimer's to assist the medical practitioners in dealing with Alzheimer's effectively [14]. To meet the Saudi Vision 2030 of using m-Health in the healthcare industry, there is a wide range of influential factors resisting the progress due to lack of exposure, awareness and appropriate skills to use the latest mobile phones in SA [15]. Different types of models are proposed so far by identifying a variety of influential factors in SA.

Therefore, in this work, a model suitable for most of the common people living in Saudi Arabia is introduced with the facility of changing language flexibility to deal with the international visitors as well. Since KSA is a pilgrimage spot in the world, observing more than a million tourists each day from across the world demands such flexibility of language in the proposed model. Most of the models introduced in recent times are dealing with health-related problems in any one direction i.e. either to deal with diabetic patients or to deal with Alzheimer patients, BP patients, etc. Most of them are quite successful but to obtain the focus of locals and international visitors the existing models seem to be facing trouble due to language barriers.

In this work, Section II deals with different types of functioning aspects of mobile health services. Different types of key systems involved in m-health systems are discussed in this section. The Section III provides the proposed method and design for the mobile health system with the events involved from different support systems, environments, healthcare systems and networking elements. A detailed discussion with the recent work areas in artificial intelligence are given in the Section IV. Finally, summarized with the complete work with the last Section $\mathrm{V}$ with conclusions.

\section{FunCtioning OF Mobile Health SERVICES}

The applications of E-Health are playing a critical role in developing countries towards improving communication between healthcare institutions and patients. They are useful to assist both the sides to manage, order, prioritize, monitor and understand the present scenario, and to react accordingly.
Blaya et al. [2] used the definition for E-Health as the "use of information and communication technologies (ICT) in support of health and health-related fields, including health-care services, health surveillance, health literature, and health education, knowledge and research" from the reports of World Health Organization (WHO) [16]. Later the same applications using mobile phones and handheld devices are considered as $\mathrm{m}$-health, provided enormous value and allowed to have multiple settings to deal with the doctors, patients, and healthcare institutions parallelly.

\section{A. Key Players of m-health Services}

The functioning of $m$-health services depends on a structured process and some of the key systems as shown in Fig. 1 include the following: a) electronic health records, b) laboratory information management system, c) pharmacy information system, d) patient registration and scheduling system, e) monitoring, evaluation, and patient tracking systems, f) clinical decision support system, g) patient reminder system, and h) research/data collection system.

a) Electronic Health Records: A very important and crucial system of the m-health system mostly managed by the doctors/clinicians/ staff with full details of patients from joining date to medical check-ups, reports, prescriptions, and precautions to be taken by a patient. Apart from that they also contain the information of doctors, nurses, and support staff that assisted the patient during their visit to the hospital.

b) Laboratory Information Management System: This plays a key role to support the administrators, doctors, and healthcare personals with the information related to laboratory activities. The required patient information at the correct time helps to make appropriate decisions to avoid any kind of mistake by the hospital authorities.

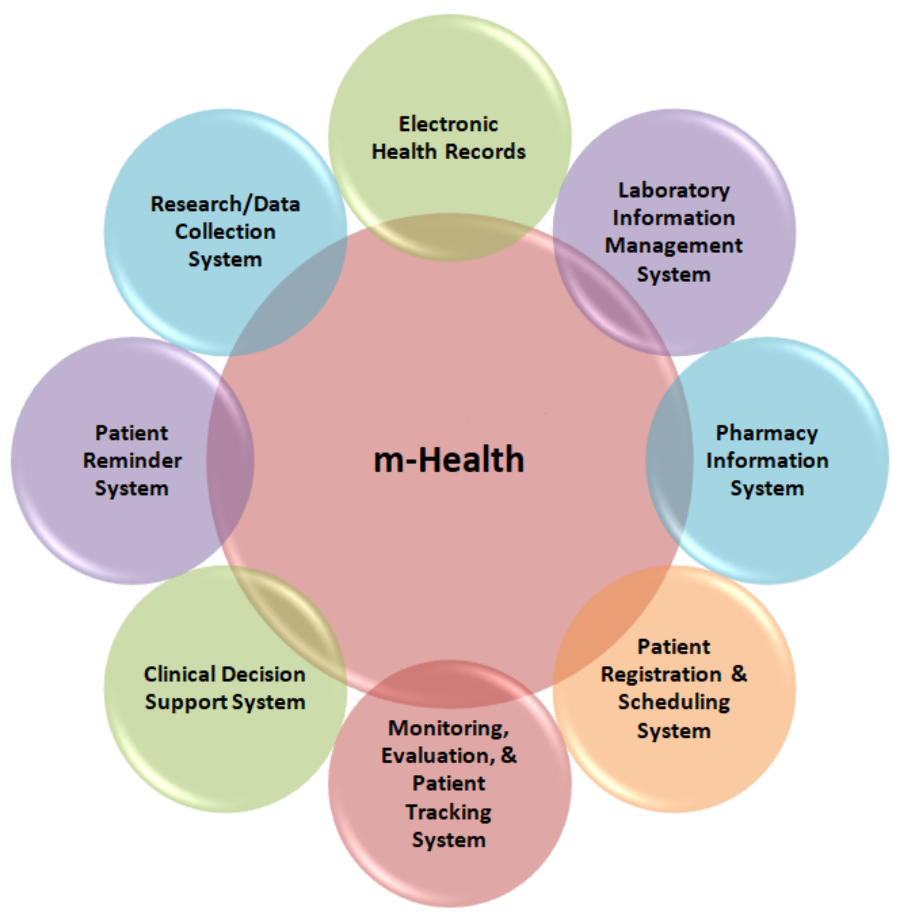

Fig. 1. Functional Parameters and Key Systems of m-Health Systems. 
c) Pharmacy Information System: This system helps the patients to order, track and dispense prescribed medications online using the m-health applications. This system is connected with a pharmacy database with a restricted set of permissions to assess only patient-related information.

d) Patient Registration and Scheduling System: The registration process helps to manage patient movement at different stages, processes and helps to maintain the census. This will help healthcare systems and even patients also to save their time by allowing both doctors and patients to get appropriate appointment timings, and relevant schedules to avoid any kind of waiting time by patients at hospital premises.

e) Monitoring, Evaluation, and Patient Tracking Systems: This system helps to aggregate information related to reporting, programs, and patient movements in the hospital to ensure that their stay in the hospital is smooth and is getting treated well in their bad health conditions.

f) Clinical Decision Support System: This system is completely based on the clinical decisions that are made earlier for a set of certain characteristics of different individual patients. The conditions or symptoms explained by a new patient are compared with the information stored in the knowledge database of the hospital and the automated algorithm-based system will generate certain recommendations to follow, as a temporary and precaution ahead of getting an appointment with the doctor.

g) Patient Reminder System: This will help the ailing patients and their family members to get alerts to perform the scheduled tasks related to medication, diagnosis, appointments, etc. This system will ensure that the patient is taking care of himself/herself with a reminder system.

h) Research/Data Collection System: The reports generated from different departments, patients, doctors, and lab technicians are going to be more useful for managing, reporting, and analyzing the same for further studies and observations in the hospital environment. These reports and collected information help to evaluate and can be used for different research programs in healthcare environment. Some of these results may help to identify key damage control decisions soon.

\section{B. Key Challenges to Implement m-health Services in Saudi Arabai}

It is a challenging task in SA to consider the evaluation of m-health towards patients with different backgrounds and ethnic groups arriving from international destinations. The challenges are linked with tele-medicines, cost-effectiveness, lack of communication skills, educational background, language barriers, etc. makes this task more complex. However, recent initiatives by the Ministry of Health-Saudi Arabia helped people to understand the importance of educating themselves with various mobile applications to obtain better healthcare services/schemes.

From the electronic health records (EHR) of different developed countries, it is observed that a) patients related with kidney problems show exemplary results; b) reduction in the clinical visits; c) larger margins of benefits for academic hospitals; d) improved efficiency of services by the network of healthcare units at different places, and e) reduced medical errors [17-19]. Therefore, it is important to introduce MHS with suitable flexibility towards local languages, global languages, sign languages for people with disabilities, and a customized module to deal with different types of technical glitches easily.

\section{Method And Design of Mobile Health System}

The proposed mobile health system (MHS) as shown in Fig. 2 follows the best suitable technologies available in the market for implementing the new mobile health system. The human body is connected with different sensors of a mobile phone installed with the developed application, to understand the body response for different activities and daily routines performed by the user. For example, the number of steps walked by a user is calculated with the in-built motion sensors. Similarly, electro encephalogram (EEG) sensors will help to understand the electrical activity of a brain and electro cardiogram (ECG) helps to record voltage versus time for the electrical activity of a heart. Whereas the galvanic skin response (GSR) helps to understand the sweat gland activity and emotional state/arousal with the help of skin response. Therefore, all these observations are monitored constantly by the mobile phone once installed with the proposed MHS app. These are communicated with the servers and databases of healthcare systems to update the patient information with different health conditions and activity records.

All these in-built sensor systems help to realize the six safety and quality principles of a healthcare system, i.e. to provide the safety, effectiveness, efficiency, timeliness, patient-centeredness, and equitableness as listed by Sannino et $a l$. [20]. The monitoring process designed in this app using a mobile phone helps realize the status of patient safety with a constant monitoring and feedback approach as shown in Fig. 2. The sensor system within the mobile phone will alert the patient if the total number of steps walked for a day or below average or very less as compared with the defined goals. The time to time information about the medication to be followed, dosage to consume, number of pills to be used, number of times the medicine to be consumed, water consumption levels, sleeping hours, etc. are provided in this application; and all these information is directly connected with the database of healthcare management for the assessment by a specialist. A predictive data analytics approach is used to define, monitor, and alert the patient by using the information obtained from the observation of mobile data.

\section{A. Proposed Healthcare Services for Saudi Arabia Environment}

The flow diagram of the events in the proposed MHS is as shown in Fig. 3 explains different activities and divided into four (4) stages as a) Patient Environment, b) Support Systems, c) Networking Systems, and d) Smart Healthcare System. 

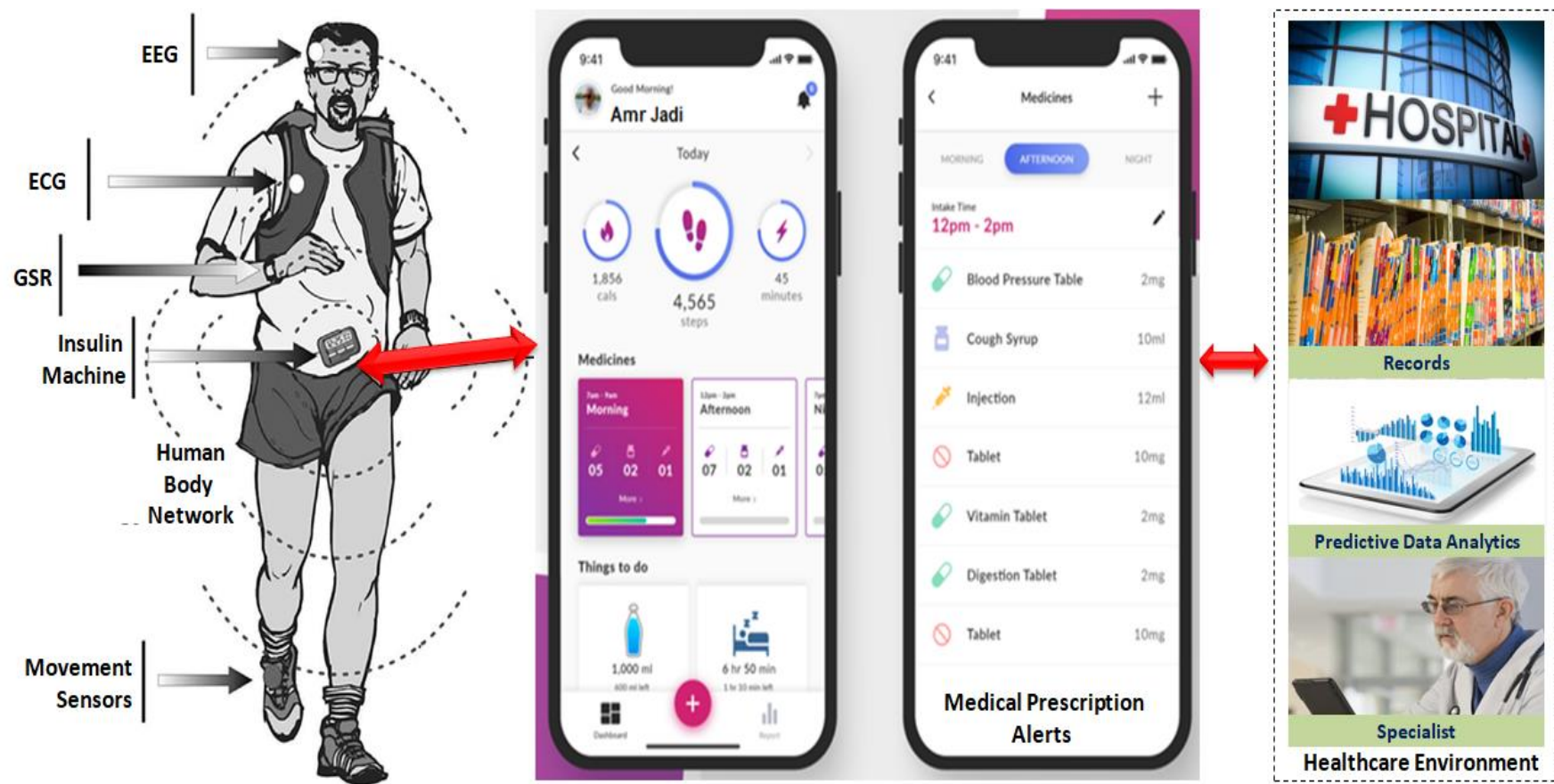

Fig. 2. Proposed Mobile Health System.

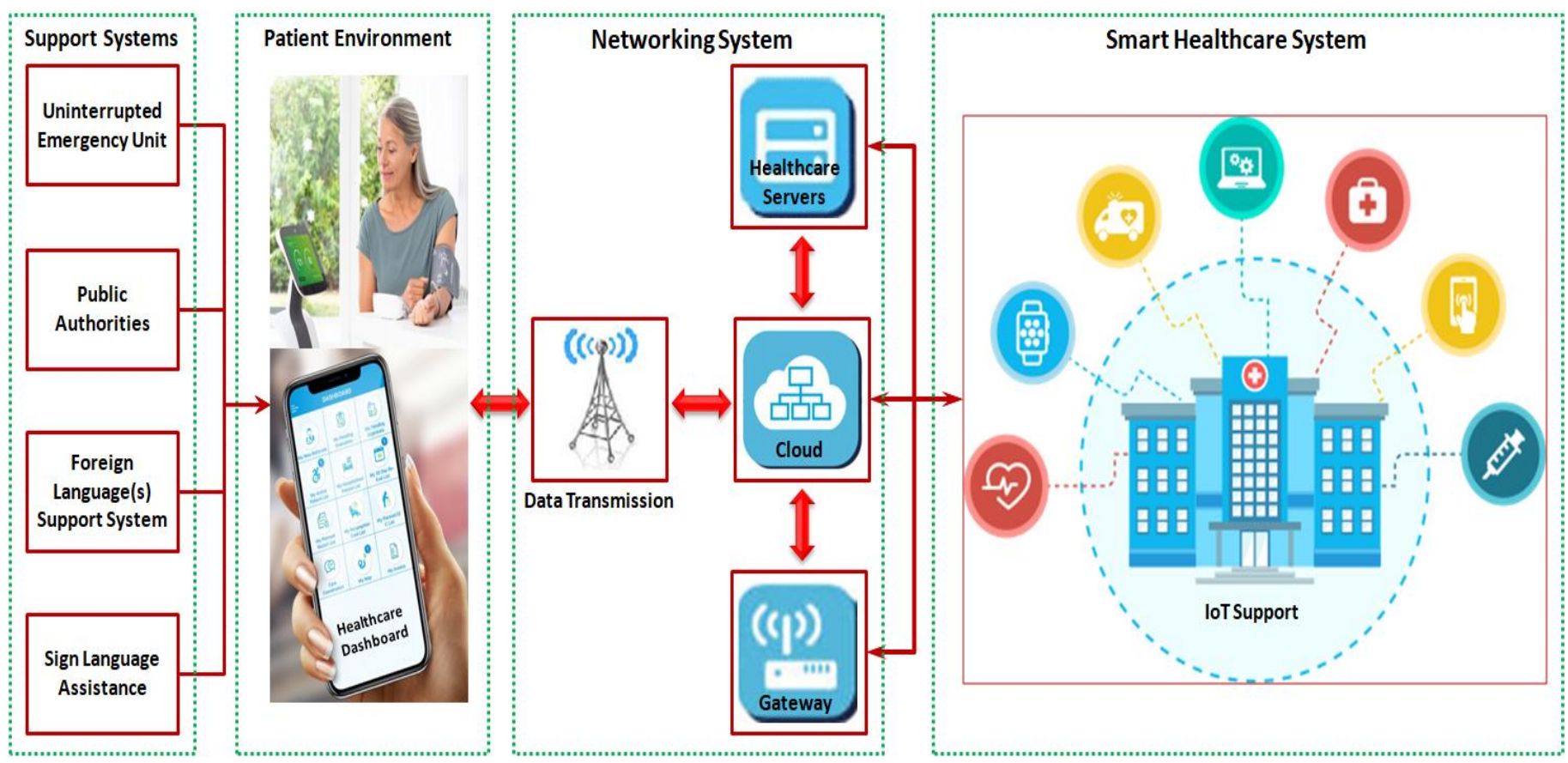

Fig. 3. Flow Diagram of Events in the Proposed Mobile Health System.

a) Patient Environment: The patient will be equipped with the needful medical arrangements at home with all the medical devices and mobile connectivity with a proper internet connection. The mobile is assumed to be a smartphone with advanced features and is installed with the proposed MHS application that has been developed to test different activities.

b) Support Systems: These systems are going to play a crucial role by providing adequate information on daily needs and keep the patient updated with different events/alerts to avoid some of the difficulties while travelling on the streets or too far end locations in Saudi Arabia. This module addressed three important aspects related to emergency services with no difficulties or delays, public authorities, foreign language support systems, and sign language assistance systems.

The emergency services are involved with police, ambulance, and fire systems with additional quickest connectivity modules are provided in this method. These modules will activate the service providers in Saudi Arabia by 
interacting with their servers by sharing the current location of a patient. The patient location is tracked by using a global positioning system (GPS) of the mobile devices. This module helps to track the traffic police density on the roads, road blockage information from the municipal boards using an alert system, and pollution levels at certain construction sites or oil refineries before starting for a walk or a drive to a longer distance.

c) Networking Systems: The networking systems are included with four important modules, such as data transmission, cloud services, network gateway, and healthcare servers. The data transmission takes place using a mobile network with $4 \mathrm{G}$ connectivity to perform the operations of the proposed MHS. This need high-speed internet services to obtain information with accuracy and quality.

A cloud system is used to interact with different healthcare systems operating on a single platform to support the patient details available in different hospitals and branch locations in different parts of the kingdom. The network servers are connected to the cloud to ensure that the patient information from different healthcare centers is being stored and allowed to use by the doctors, experts, and patients on demand.

d) Smart Healthcare System: The complete hospital environment is connected digitally with a variety of internet of things (IoT) devices and equipment to establish a smart environment in the healthcare system. The connectivity of IoT helps to increase the access for information and accuracy of dealing with patients improves at a quicker rate. This, in turn, helps the doctors and experts to respond quickly to the inpatients and out-patients with the collective response from the server's information on their computer screen with all feedbacks and updated information.

\section{B. Key Features of the Proposed Method}

- This system helps the people of SA to get suitable assistance and feedback from the healthcare system at their doorstep.

- The health condition of the patients is monitored by the in-built sensors of the mobile devices from the proposed MHS and communicates constantly with the doctors and experts of the healthcare system.

- For any kind of emergency calling the patients can press the emergency symbol that is available on all screens of the app with a quick response.

- The support of public authorities is provided constantly using updated alerts once the patient is trying to use any kind of traveller's apps, Google maps, etc. to search for a location.

- The foreigners visiting SA are supported with the inbuilt language options for the global languages using a simple button press. On the other side, sign languages are also provided for the convenience of people to understand if they cannot understand any languages, which are listed in the application.
- The internet connectivity is going to play a vital role in this system and needs $4 \mathrm{G}$ networks with high-speed data processing networking set up to establish the connectivity with cloud systems and IoT supported healthcare systems.

- The IoT devices are connected with good internet connectivity and are linked with the healthcare servers to update the events between different departments, doctors, doctors and patients, patients and lab technicians, etc.

- All these reports generated from different resources are analyzed by using the predictive data analytics for faster assessment of the reports and analyze the current conditions effectively. So far by using the NN and AIbased predictive system in the proposed MHS, the accuracy of $95 \%$ is achieved for some of the healthrelated diseases/problems.

\section{DISCUSSIONS}

Usage of artificial intelligence (AI) for clinical trials is considered in this module for the analysis of patient conditions at different levels using the information obtained from the IoT devices. Usage of neural networks (NN) and $\mathrm{AI}$ is experimented in many medical applications to design different types of risk management systems [21, 22]. Similar applications can be used here for the proposed MHS, which helps to assess the risks related to blood pressure (BP), sugar levels and heartbeats of the patients at different locations. The modules used in the previous work were extended by the author to implement the proposed system using $\mathrm{NN}$ to enhance the speed of risk prediction and risk mitigation [23]. Similar modules are used and implemented with extended IoT arrangements to test the set up for wide-range coverage of patients and healthcare systems.

Larger sections of clinical professionals are suggesting a positive growth using m-health technologies and are expressing a higher rate of satisfaction across various parts of the globe. A research report of Burrows revealed that $53 \%$ of the clinical trials are having the biggest impact because of mhealth or AI [24]. The likeliness of increasing usage of mhealth in the coming years is most promising and $79 \%$ of the respondents in his research show positive intent for m-health usage in the healthcare industry. The accuracy obtained from the proposed system towards risk prediction is around $95 \%$ concerning BP, Sugar and heartbeat values. Also, it is seen that the IoT based applications are playing a key role in reducing the overall expenditure (near to 30 to 35 percent) for the patients and hospital management. Most of the time by using IoT it is seen that reduced patient visits, reduced diversion of doctor's and lab technician's attention during the working hours, reduced waiting hours for getting pharmacy billing, etc. With these advantages, the proposed MHS improved the overall performance to a satisfactory level by using m-health services.

Presently the proposed MHS application is installed within a single healthcare unit including their patients, doctors, technicians, and users to test with different possibilities. Huge data records generated by doctors, patient reports, etc. are 
analyzed using bigdata techniques as discussed by Mishra and Chakraborty [25]. The proposed system is under the testing generates accurate results towards risk prediction and risk mitigation with the limited data.

\section{CONCLUSIONS}

From the obtained results of this work, the quality of the healthcare services can be improved and the overall satisfaction levels of the patients can be taken care of by using the proposed MHS. Extremes situations can be avoided by supporting the patient with real-time monitoring, guidance, and medical advice for any kind of unfortunate event using a mobile app helps to deal with patient emotionally. Getting panic is the tendency of a patient with no adequate information at hand can be solved and a communication medium can be established by introducing mobile health services. The proposed approach is capable of solving the language barriers effectively and it can also help the people with speaking and listening disabilities as well. The accuracy of risk prediction and mitigation using the proposed EHS found to be $95 \%$, which is a good improvement as compared with the existing m-health service providers. Applications of bigdata and AI are being tested for various IoT operations for creating a smart healthcare environment.

\section{ACKNOWLEDGMENT}

The authors gratefully acknowledge the support and facilities provided by the Management and Department of Computer Science and Information, University of Hail, Hail, Saudi Arabia.

\section{REFERENCES}

[1] P. Cipresso, S. Serino, D. Villani, C. Repetto, L. Sellitti, G. Albani, A. Mauro, A. Gaggioli, and G. Riva. "Is your phone so smart to affect your state? An exploratory study based on psychophysiological measures." Neurocomputing 84 (2012): 23-30.

[2] J. A. Blaya, H. S. Fraser, and B. Holt. "E-health technologies show promise in developing countries." Health Affairs 29, no. 2 (2010): 244251.

[3] S. M. Edworthy. "Telemedicine in developing countries: may have more impact than in developed countries." (2001): 524-525.

[4] Arab News. M-health technologies to be key enablers of Saudi digital transformation process: Expert. [Online] March 13, 2019 Accessed on [January 12, 2020].

[5] E. M. Borycki, F. M. S. Househ, A. W. Kushniruk, C. Nohr, and H. Takeda. "Empowering patients: making health information and systems safer for patients and the public." Yearbook of medical informatics 21, no. 01 (2012): 56-64.

[6] A. Al-Harbi. "Healthcare providers' perceptions towards health information applications at King Abdul-Aziz Medical City, Saudi Arabia." International Journal of Advanced Computer Science and Applications 2, no. 10 (2011): 10-13.

[7] A. Paules Ciprés, H. M. Fardoun, D. M. Alghazzawi, and M. Oadah. "KAU e-health mobile system." In Proceedings of the 13th International Conference on Interacción Persona-Ordenador, pp. 1-5. 2012.

[8] K. A. Al-Rubeaan, A. M. Youssef, S. N. Subhani, N. A. Ahmad, A. H. Al-Sharqawi, and H. M. Ibrahim. "A Web-based interactive diabetes registry for health care management and planning in Saudi Arabia." Journal of medical Internet research 15, no. 9 (2013): e202.
[9] R. S. Istepanian, A. Mousa, N. Haddad, A. Sungoor, T. Hammadan, H. Soran, and T. Al-Anzi. "The potential of m-health systems for diabetes management in post conflict regions a case study from Iraq." In 2014 36th Annual International Conference of the IEEE Engineering in Medicine and Biology Society, pp. 3650-3653. IEEE, 2014.

[10] B. M. Silva, J. J. Rodrigues, I. de la Torre Díez, M. López-Coronado, and K. Saleem. "Mobile-health: A review of current state in 2015." Journal of biomedical informatics 56 (2015): 265-272.

[11] M. Alotaibi, and I. Choudhury. "A social robotics children diabetes management and educational system for Saudi Arabia: system architecture." In 2015 Second International Conference on Computer Science, Computer Engineering, and Social Media (CSCESM), pp. 170174. IEEE, 2015.

[12] S. H. Almotiri, M. A. Khan, and M. A. Alghamdi. "Mobile health (mhealth) system in the context of IoT." In 2016 IEEE 4th international conference on future internet of things and cloud workshops (FiCloudW), pp. 39-42. IEEE, 2016.

[13] S. A. AlKlayb, M. K. Assery, A. AlQahtani, M. AlAnazi, and S. C. Pani. "Comparison of the effectiveness of a mobile phone-based education program in educating mothers as oral health providers in two regions of Saudi Arabia." Journal of International Society of Preventive \& Community Dentistry 7, no. 3 (2017): 110.

[14] A. O. Elfaki, and M. Alotaibi. "The role of M-health applications in the fight against Alzheimer's: current and future directions." Mhealth 4 (2018).

[15] N. Aljohani, and D. Chandran. "Adoption of M-Health Applications: The Saudi Arabian Healthcare Perspectives." Australian Conference on Information Systems 2019, Perth: 180-186.

[16] World Health Organization. "58th World Health Assembly Report; 1625 May 2005." Geneva: WHO (2005).

[17] T. Garrido, L. Jamieson, Y. Zhou, A. Wiesenthal, and L. Liang. "Effect of electronic health records in ambulatory care: retrospective, serial, cross sectional study." Bmj 330, no. 7491 (2005): 581.

[18] D. C. Evans, W. P. Nichol, and J. B. Perlin. "Effect of the implementation of an enterprise-wide Electronic Health Record on productivity in the Veterans Health Administration." Health Economics, Policy and Law 1, no. 2 (2006): 163-169.

[19] V. E. Pollak, and J. A. Lorch. "Effect of electronic patient record use on mortality in End Stage Renal Disease, a model chronic disease: retrospective analysis of 9 years of prospectively collected data." BMC medical informatics and decision making 7, no. 1 (2007): 38 .

[20] G. Sannino, G. De Pietro, and L. Verde. "Healthcare Systems: An Overview of the Most Important Aspects of Current and Future mHealth Applications." In Connected Health in Smart Cities, pp. 213-231. Springer, Cham, 2020.

[21] A. Jadi, H. Zedan, and T. Alghamdi. "Risk management based early warning system for healthcare industry." In 2013 International Conference on Computer Medical Applications (ICCMA), pp. 1-6. IEEE, 2013.

[22] A. Jadi. "Improving the Emergency Services for Accident Care in Saudi Arabia." International Journal of Advanced Computer Science and Applications 7, no. 9 (2016).

[23] A. Jadi. "An Early Warning System for Risk Management." Ph.D Thesis, DeMontfort University (2013).

[24] A. Burrows. "MHealth in clinical trials: $79 \%$ of companies increasing usage - data report analysis. [Online] available at URL: $<$ https://informaconnect.com/mhealth-clinical-trials-data-reportanalysis/>, [accessed on February 12, 2020].

[25] K. N. Mishra, and C. Chakraborty. "A Novel Approach Towards Using Big Data and IoT for Improving the Efficiency of m-Health Systems." In Advanced Computational Intelligence Techniques for Virtual Reality in Healthcare, pp. 123-139. Springer, Cham, 2020. 